Popular Summary

Performance of Goddard Earth Observing System GCM Column Radiation Models under Heterogeneous Cloud Conditions

L. Oreopoulos ${ }^{\mathrm{a}, \mathrm{b}}$, M.-D. Chou ${ }^{\mathrm{b}}$, M. Khairoutdinov ${ }^{\mathrm{c}}$, H. W. Barker ${ }^{\mathrm{d}}$, and R. F. Cahalan ${ }^{\mathrm{b}, \mathrm{a}}$

a. JCET-University of Maryland Baltimore County, Baltimore, MD

b. Laboratory for Atmospheres, NASA Goddard Space Flight Center, Greenbelt, MD

c. Dept. of Atmospheric Sciences, Colorado State University, Fort Collins, CO

d. Meteorological Service of Canada, Downsview, ON

Submitted for a special issue of "Atmospheric Research", July 31, 2003

Global Climate Models (GCMs) are one of our most valuable tools in climate studies, including prediction of future climatic states. One of the most important components of these models is their Column Radiation Models (CORAMs) which calculate the interactions of atmospheric constituents with solar and terrestrial radiation. CORAMs have to make many simplifications in the way they perform calculations because they compete for computational power with other components of the GCM and because of incomplete knowledge of the atmospheric state, especially cloud structure. In this paper, a unique global single-day dataset produced by NCAR's GCM with a 2-D Cloud Resolving Model installed in each gridbox is available for testing GSFC CORAMs by Chou and collaborators. The dataset is unique in the sense that information on the structure of clouds within a coarse GCM gridbox is known and can be used for reference ("truth") calculations. We find that the original solar and infrared GSFC CORAMs performs quite well compared to the reference calculations and that the performance of the solar CORAM improves further by introducing a recently suggested scheme that implicitly accounts for cloud inhomogeneity. These results suggest that previous studies demonstrating the inaccuracy of plane-parallel models may have unfairly focused on worst scenario cases, and that current radiative transfer algorithms of GCMs may be more capable than previously thought in estimating realistic spatial and temporal averages of radiative fluxes, as long as they are provided with correct mean cloud profiles. However, even if the errors of the particular CORAMs are small, they seem to be systematic, and the impact of the biases can be fully assessed only with GCM climate simulations. 


\section{Performance of Goddard Earth Observing System GCM Column Radiation Models under heterogeneous cloud conditions}

L. Oreopoulos ${ }^{\mathrm{a}, \mathrm{b}}$, M.-D. Chou ${ }^{\mathrm{b}}$, M. Khairoutdinov ${ }^{\mathrm{c}}$, H. W. Barker ${ }^{\mathrm{d}}$, and R. F. Cahalan ${ }^{\mathrm{b}, \mathrm{a}}$ Submitted for a special issue of "Atmospheric Research", July 31, 2003

a. JCET-University of Maryland Baltimore County, Baltimore, MD

b. Laboratory for Atmospheres, NASA Goddard Space Flight Center, Greenbelt, MD

c. Dept. of Atmospheric Sciences, Colorado State University, Fort Collins, CO

d. Meteorological Service of Canada, Downsview, ON 


\begin{abstract}
We test the performance of the shortwave (SW) and longwave (LW) Column Radiation Models (CORAMs) of Chou and collaborators with heterogeneous cloud fields from a global sigle-day dataset produced by NCAR's Community Atmospheric Model with a 2-D CRM installed in each gridbox. The original SW version of the CORAM performs quite well compared to reference Independent Column Approximation (ICA) calculations for boundary fluxes, largely due to the success of a combined overlap and cloud scaling parameterization scheme. The absolute magnitude of errors relative to ICA are even smaller for the LW CORAM which applies similar overlap. The vertical distribution of heating and cooling within the atmosphere is also simulated quite well with daily-averaged zonal errors always below $0.3 \mathrm{~K} / \mathrm{d}$ for $\mathrm{SW}$ heating rates and $0.6 \mathrm{~K} / \mathrm{d}$ for LW cooling rates. The SW CORAM's performance improves by introducing a scheme that accounts for cloud inhomogeneity.

These results suggest that previous studies demonstrating the inaccuracy of planeparallel models may have unfairly focused on worst scenario cases, and that current radiative transfer algorithms of General Circulation Models (GCMs) may be more capable than previously thought in estimating realistic spatial and temporal averages of radiative fluxes, as long as they are provided with correct mean cloud profiles. However, even if the errors of the particular CORAMs are small, they seem to be systematic, and the impact of the biases can be fully assessed only with GCM climate simulations.
\end{abstract}




\section{Introduction}

There have been numerous efforts during the past few years to quantify the effects of cloud water horizontal inhomogeneity on the radiative properties of clouds. Most of these efforts have concentrated on solar wavelengths which suffer greater errors than infrared wavelengths where cloud emissivity saturates at relatively low values of cloud optical depth. In a recent paper Rossow et al. (2002) performed a literature survey which revealed that the shortwave (SW) albedo bias estimates from neglecting horizontal inhomogeneity in radiative calculations had a wide range of 0.025 to 0.3 in various observational studies. This range does not correspond to global averages, but values estimated under a variety of conditions and for different cloud types. Given the large SW radiative impact of horizontal heterogeneities it was only logical that research efforts would be directed to develop algorithms that account for them in modeling applications and are as simple as possible, ideally following plane-parallel formalisms. Clearly, the algorithms developed with single-layer cloud systems in mind (Cahalan et al. 1994; Barker 1996; Cairns et al., 2000; Szczap et al. 2000a,b), were not suitable for General Circulation Models (GCMs), which use multi-layer algorithms that are not necessarily straightforward extensions of their single-layer counterparts. The first complete inhomogeneous solar algorithm conforming to GCM requirements was developed by Oreopoulos and Barker (1999). Modifications and improvoments of the basic concepts of this algorithm in terms of computational speed and concurrent treatment of vertical cloud variability followed in publications by Barker and $\mathrm{Fu}(2000)$ and Kato (2003). During the same time, algorithms that take into account the inhomogeneous nature of clouds at the longwave (LW) part of the spectrum were also developed (Li and Barker, 2002). 
The main purpose of this paper is to present an evaluation of the performance under inhomogeneous cloud conditions of two Column Radiation Models (CORAMs) developed by Chou and collaborators (see references), and used in various NASAGoddard GCMs. Since, however, the cloud statistics needed for input are derived from the same cloud distributions used in our reference ("truth") calculations, the errors presented in the following are not in any way typical of the radiation budget errors that would arise in GCM simulations using these CORAMs; they are actually typical errors that would arise if perfect cloud statistics, suited to the input needs of the particular CORAMs, were used. Extended tests with more sophisticated research versions of the CORAM that account for cloud inhomogeneity is not a top priority in this study, but some relevant findings will be shown for the SW CORAM (which takes the lion's share of our analysis). The CORAMs are evaluated on global scales, but with the caveat that the input cloud data come from only one simulated day. Still, this is an improvement over previous studies where assessments of the errors of plane-parallel homogeneous (PPH) codes were performed using a limited number of cloud fields generated either by theoretical fractal models (e.g. Cahalan et al., 1994), or by Cloud Resolving Models (CRMs) attempting to simulate clouds of only a particular type (e.g. Barker et al., 1999; Barker and Fu, 1999), or clouds of a specific field campaign (Fu et al., 2000).

We present a description of the input data set in section 2 , a description of the CORAMs in section 3, and results from the SW and LW experiments in sections 4 and 5 , respectively. We close with a discussion of our findings in section 6 . 


\section{Dataset: CAM/CRM clouds}

Recently, Khairoutdinov et al. (2003) embedded a 2-D version of the 3-D CRM described in detail by Khairoutdinov and Randall (2003) into each grid column of NCAR's Community Atmosphere Model (CAM, the atmospheric component of the Community Climate System Model GCM) version 1.8, to serve as a super-parameterization of clouds. (for earlier implementations of this concept see Khairoutdinov and Randall, 2001). We have selected a single day (January 1) from a 500-day long simulation with the superparameterization, initialized on September $1^{\text {st }}$, and using T42 resolution $\left(2.8^{\circ} \times 2.8^{\circ}\right.$ grid) with 26 vertical layers (24 for the CRM itself, at the same levels as the lowest 24 layers of the CAM). The cloud fields used as input in our radiative transfer calculations with the CORAMs come from 24 global "snapshots" saved at 1-hour intervals for this day. Some snapshots from this day were also used in a recent study by Räisänen et al. (2003). Within each of the $64 \times 128$ gridboxes covering the globe, clouds are then resolved in 64 columns, of $4 \mathrm{~km}$ horizontal width (aligned in the west-east direction) and 24 vertical layers.

Figure 1 shows latitude-height (zonally-averaged) cross-sections of daily-averaged (averaged over 24 snapshots) cloud properties for each model layer: cloud fraction, (total) water path (liquid water path + ice water path), and cloud inhomogeneity parameter $v$ of Barker (1996) calculated from the method of moments (MOM) as the square of the ratio of mean to standard deviation of the water path of each layer. These figures show that the super parameterization within the CAM captures the main contrast in cloud types between tropics and mid-latitudes: clouds in the tropics are higher in the troposphere, thicker, and more heterogeneous (smaller values of $v$ ). Khairoutdinov et al. (2003) further discuss the degree realism of numerous output fields from the entire experiment. 


\section{The Column Radiation Models}

We use both the SW and LW CORAMs developed by M.-D. Chou and collaborators and used in NASA-GSFC GCMs. The latest versions of the codes can be downloaded from the World Wide Web address http://climate.gsfc.nasa.gov/ chou. This website also provides the accompanying NASA technical documents that describe the CORAMs (Chou and Suarez, 1999; Chou et al., 2001). For the SW CORAM the reader can also refer to Chou et al. (1998).

Some of the most recent changes in the SW CORAM are the inclusion of variable cloud particle size $\left(r_{\mathrm{e}}\right)$ that depends on water content, following McFarquhar (2001) for ice clouds, and Szczodrak et al. (2001) for water clouds, and of a new parameterization for ice particle single-scattering albedo (Chou et al., 2002). We will discuss the implications of the first of these changes in our calculations in the following section. An important feature of the SW CORAM is the cloud overlap treatment which is schematically presented in Fig. 2. Clouds are grouped into three categories: low, middle and high, separated at 700 and $400 \mathrm{hPa}$. Through two separate optical depth adjustments $\left(\tau \rightarrow \tau_{\mathrm{dd}}, \tau \rightarrow \tau_{\mathrm{df}}\right)$

$$
\begin{aligned}
& \tau_{\mathrm{dd}}=\chi_{\mathrm{dd}} \tau \\
& \tau_{\mathrm{df}}=\chi_{\mathrm{df}} \tau \\
& R_{\mathrm{dd}}\left(\tau_{\mathrm{dd}}, r_{\mathrm{e}}, \mu_{0}\right)=q R_{\mathrm{dd}}\left(\tau, r_{\mathrm{e}}, \mu_{0}\right) \\
& R_{\mathrm{df}}\left(\tau_{\mathrm{df}}, r_{\mathrm{e}}\right)=q R_{\mathrm{df}}\left(\tau, r_{\mathrm{e}}\right) \\
& q=C_{\mathrm{i}} / C_{\mathrm{j}, \max }
\end{aligned}
$$


which preserve the layer albedo $\left(R_{\mathrm{dd}}, R_{\mathrm{df}}\right)$ for direct $(d d)$ and diffuse $(d f)$ incident radiation, the cloud in any layer $i$ that has smaller cloud fraction $\left(C_{\mathrm{i}}\right)$ than the maximum cloud fraction within its group $j\left(C_{\mathrm{j}, \max }\right)$, is "stretched" and "thinned" so that it acquires the maximum cloud fraction value (for details see e.g., Chou et al., 1998). The adjustment parameters $\chi_{\mathrm{dd}}$ and $\chi_{\mathrm{df}}$ are functions of $q, \tau$, and (in the case of $\chi_{\mathrm{dd}}$ ) the cosine of the solar zenith angle (SZA), $\mu_{0}$. Once this adjustment is made, each cloud group has its own unique cloud fraction $C_{\mathrm{j}, \max }$, and clouds among different groups are assumed to overlap randomly. Clearly, the concept is inspired from the observations of Tian and Curry (1989) that neighboring clouds tend to overlap maximally while clouds separated by clear skies tend to overlap randomly. This overlap assumption allows each gridbox to be divided into $\leq 2^{\mathrm{j}}$ sub-columns $(j \leq 3)$ where each layer is either completely cloudy or cloud-free (Fig. 2, right), and for which the full vertical distribution of fluxes can be calculated. The weighted average of the sub-column estimates gives the mean for the entire gridbox, and can be thought of as a "crude" Independent Column Approximation (ICA).

For LW calculations the concept of the probability of clear line-of-sight of Harshvardhan et al. (1987) is used to calculate the flux transmittance between two different layers. Clouds are again grouped in three different height ranges, as in Fig. 2, with maximum overlap assumed within each group, and random overlap between groups. Probabilities of clear line-of-sight are calculated for each group, and the total probability for any two levels is the product of probabilities of the groups contained within these levels. For details see Chou et al. (2001).

\section{SW experiments}

For our broadband SW calculations we performed four different sets of runs: two sets with the original version of the CORAM and two with modified versions. Each set 
consisted of runs for every gridbox which received non-zero solar illumination calculated according to its center latitude and longitude coordinates and according to the Greenwich Mean Time (GMT) corresponding to a particular snapshot. The original version of the CORAM was used for ICA and PPH runs. The ICA runs were performed as follows: the CORAM was run for each of the 64 sub-columns of the gridbox with the cloud fraction of each layer of a sub-column either 0 or 1 and with a particle size profile that depended on the water content profile according to McFarquhar (2001) and Szcodrak (2001); the gridbox-mean (ICA) fluxes were derived by averaging the results for the 64 columns. Note that the discussion pertaining to Fig. 2 becomes irrelevant for ICA runs because of the binary nature of layer cloud fraction. The PPH runs were performed as follows: for each vertical layer the cloud fraction, and the mean liquid and ice water paths were determined for the gridbox; the particle sizes of each phase were estimated from the individual mean water paths; the CORAM in its original form was applied to each gridbox using as input these cloud fraction, water path and particle size profiles.

One of the modified versions of the CORAM, called "PPH random" was designed to reveal the impact of the cloud adjustment and overlap assumption depicted in Fig. 2. In this PPH variant, the albedo and transmittance of a layer $i$ was calculated as the weighted average of the corresponding clear and cloudy fractional fluxes:

$$
f_{\mathrm{i}}=\left(1-C_{\mathrm{i}}\right) f_{\mathrm{clr}, \mathrm{i}}+C_{\mathrm{i}} f_{\mathrm{cld}, \mathrm{i}}
$$

where $f_{\mathrm{i}}, f_{\mathrm{clr}, \mathrm{i}}, f_{\mathrm{cld}, \mathrm{i}}$ are one of albedo, diffuse transmittance and direct transmittance of the entire layer $i$ and of the clear and cloudy parts, respectively. The flux profile was then 
determined by radiatively linking the $f_{\mathrm{i}}$ 's (see eq, 6.9-6.16 in Chou and Suarez, 1999). As explained in Oreopoulos and Barker (1999) and Barker et al. (1999), because this type of linking does not contain any information on whether the flux transmitted and reflected to a layer comes from the clear or cloudy parts of the layers above and below, it is in practice equivalent to an indiscriminate application of the random overlap assumption for all layers, regardless of whether they are contiguous or not.

The other modified version is based on the Gamma Weighted Two Stream Approximation (GWTSA) of Oreopoulos and Barker (1999) where the direct beam and $\delta$-Eddington $\mathrm{PPH}$ solutions $f_{\mathrm{PPH}, \mathrm{i}}$ of layer $i$ are replaced by their counterpart analytic solutions $f_{\Gamma, i}$ obtained by integrating over a gamma distribution of optical depths $p_{\Gamma}(\tau)$ with mean optical depth $\bar{\tau}$ and variability parameter $v=(\bar{\tau} / \sigma)^{2}(\sigma$ is the standard deviation of the optical depth distribution):

$$
\begin{aligned}
& f_{\Gamma, i}=\int_{0}^{\infty} p_{\Gamma}(\tau) f_{P P H, i} \mathrm{~d} \tau \\
& p_{\Gamma}(\tau)=\frac{1}{\Gamma(v)}\left(\frac{v}{\bar{\tau}}\right)^{\nu} \tau^{\nu-1} e^{-v \tau / \bar{\tau}}
\end{aligned}
$$

$\Gamma(v)$ is the gamma function. The solutions of (3a) for reflectance, diffuse transmittance and direct transmittance can be found in Appendix A of Oreopoulos and Barker (1999). Whenever skies are clear, the old $\delta$-Eddington PPH solutions are invoked for direct incidence. For diffuse incidence on clear skies, the PPH solutions of Meador and Weaver (1980) are implemented, replacing the previously used empirical approximation of setting the SZA to $53^{\circ}$ in the solutions for direct incidence (Chou and Suarez, 1999). 
The actual implementation in the Goddard SW CORAM differes from the one described by Oreopoulos and Barker (1999) in several ways: first, the scaling of optical depth conveyed by eq. (1) is retained; second the cloud optical depth adjustment described in their paper by eq. (21-22) is omitted; third, MOM estimates of $v$ are used instead of maximum likelihood estimates; and fourth, $v$ is estimated from the water path variability. This value of $v$ is not identical to the value derived from the optical depth variability, as was the case in Oreopoulos and Barker (1999) who used a constant particle size assumption. Estimating $v$ from the water path variability is an approximation that simplifies matters since the spectral dependence of optical depth does not carry over in the calculations as spectral dependence of $v$. It is also compatible with possible future implementations of GWTSA in GCMs which are expected to be able to predict subgrid water path variability, but not subgrid variable of particle size (unless a constant droplet number density assumption is invoked).

All the results that follow are averaged over the 24 snapshots (daily averages), and the rms errors relative to the ICA are calculated using these daily averages, i.e. the rms errors encompass only spatial and not temporal differences. As mentioned before, our runs used a realistic global distribution of SZAs for each snapshot corresponding to January 1 , so we can express the SW CORAM performance results in energy units $\mathrm{Wm}^{-2}$. The SW surface albedo was assumed to be spectrally independent and invariable to SZA variations or the presence of clouds (which largely regulate the relative amounts of direct to diffuse solar irradiance reaching the ground). The broadband albedo values from a February 1-5 simulation of the Meteo France ARPEGE GCM were used (Räisänen, 1999). Aerosols were neglected, and the ozone profile of a standard midlatitude summer 
atmosphere was used for all gridboxes. ICA is our reference ("truth") estimate. Barker et al. (1999) and others have shown than the domain average errors of the ICA relative to a full 3D Monte Carlo calculations are small for most cloud fields.

Figure 3 shows the global mean errors relative to the ICA for the three different versions of the SW CORAM and for the following quantities: reflected flux at the top-ofthe-atmosphere (RTOA), net flux absorbed at the surface (NSFC), and net flux absorbed within the atmosphere (ATMA). Averages are shown for all gridboxes as well as gridboxes that are overcast on a daily-averaged basis ( 1500 gridboxes, "ovc"). Errors in RTOA and NSFC are of opposite sign (positive sign indicates overestimate relative to ICA), as expected, and of about equal magnitude. Consequently, mean errors in ATMA are very small. PPH overestimates global RTOA by $\sim 4 \mathrm{Wm}^{-2}$, PPH random increases the error by about $50 \%$, and GWTSA reduces it by about the same amount, while changing the sign of the error. Interestingly, when only overcast gridboxes are considered, the GWTSA performance improves for RTOA and NSFC, in contrast to PPH and PPH random for which it deteriorates. Overall, however, the errors are far smaller than previously reported in studies of individual cloud systems (Barker et al., 1999; Oreopoulos and Barker 1999; Barker and Fu, 1999).

The PPH error increases only modestly when gridboxes with either thick or strongly inhomogeneous clouds are averaged separately. This is shown in Fig. 4 which summarizes global average (also properly area-weighted) errors for gridboxes with mean (over cloudy sub-columns only) integrated water path (daily-averaged) greater than 200 $\operatorname{gm}^{-2}(\sim 2100$ gridboxes) and gridboxes with $v$ of integrated water path less than 1.5 ( 2600 gridboxes). The difference between PPH and PPH random is, however, much 
larger than in Fig. 3. GWTSA reduces the errors significantly for this particular subset of strongly inhomogeneous clouds, but has a smaller impact for the thick cloud subset.

The next point of interest would naturally be the geographical distribution of errors. The latitudinal behavior is highlighted in Fig. 5 which shows the zonally-averaged mean and rms errors for RTOA. As before, prior daily-averaging has been applied, and negative mean error values indicate underestimate relative to the ICA. It is immediately apparent that the latitudinal variation of PPH and PPH random errors is consistent with the cloud properties shown in Fig. 1. Thick and inhomogeneous clouds just south of the equator result in PPH errors approaching $10 \mathrm{Wm}^{-2}$, which GWTSA reduces by a factor of almost 5. Around $5^{\circ} \mathrm{N}$, the PPH error has a local minimum, and the GWTSA a local maximum, but for the PPH this is largely a result of error cancellation as the bottom panel (rms errors) reveals. Since the errors are expressed in flux units of $\mathrm{Wm}^{-2}$, the amount of available solar insolation plays a crucial role in determining the magnitude. Thus, we see much greater errors in the mid-latitudes of the southern hemisphere ( $\mathrm{SH}$ ) than the mid-latitudes of the northern hemisphere $(\mathrm{NH})$, despite the fact that the cloud water path and inhomogeneity are only moderately higher in the SH.

Some interesting conclusions can be drawn by also studying the distribution of errors for individual gridboxes (no geographic averaging). Fig. 6, for example, shows the daily-averaged RTOA errors of all gridboxes by plotting PPH random and GWTSA errors vs. the PPH errors. We see that the PPH error can occasionally be negative (PPH underestimates TOA reflected flux) which seems to conflict the well-ingrained perception that PPH always overestimates the albedo of inhomogeneous cloudy atmospheres. The reason that PPH underestimates are encountered in our results is that we are dealing with 
a multi-layer atmosphere with many layers partially filled with clouds so that the scalings of eq. (1) are often invoked. The final cloud fraction and optical depth profile that is used as input into the PPH run can produce smaller reflected and larger transmitted fluxes than the ICA run for which the exact optical depth and relative vertical position of clouds is known. When the PPH underestimates, it performs always better than the GWTSA (points below the diagonal in the lower left quadrant). The reason is that the current version of the CORAM with GWTSA keeps the scalings of the original PPH version, while also reducing the albedo of each cloudy layer by replacing the two-stream PPH solutions with their counterpart GWTSA solutions. On the other hand, the PPH random can change the values of the flux profile so drastically that the sign of the error reverses (upper two quadrants). Indeed, it is extremely rare that the PPH random will underestimate the reflectance since the indiscriminate application of random overlap almost always overestimates cloud fraction, and thus maximizes the amount of clouds exposed to downward diffuse and direct fluxes. Thus, as Fig. 6 illustrates, occurences of positive PPH random errors are extremely rare (lower two quadrants). When the PPH overestimates (upper and lower right quadrants), the GWTSA can either overestimate (upper right quadrant) or underestimate (lower right quadrant); in the first case, the GWTSA performs at most times better than PPH (points below the diagonal) since the GWTSA two stream solutions give lower values of albedo (smaller overestimates); in the second case the GWTSA overcorrects for gridboxes where PPH errors are close to zero, and improves results only as positive PPH errors grow larger.

Shifting our focus now to the distribution of fluxes within the atmosphere, Fig. 7 shows the global daily-averaged SW heating rates (HRs) for the four sets of runs (left) as 
well as the rms errors relative to ICA of the three approximations (right). Both panels clearly demonstrate the fact that the distribution of heating within the atmosphere is captured quite nicely by all three approximations. This is consistent with the small values of ATMA global mean error shown previously in Fig. 3. Still, the overestimation of heating at the lower layers of the troposphere by GWTSA is evident and this is due to the overestimation of flux transmitted through the cloudy layers above. The opposite happens for PPH and PPH random. GWTSA is performing worse than the PPHs in the higher levels of the atmosphere. Fig. 8 shows that GWTSA actually overestimates the heating consistently at all latitudes (max. value: $\sim 0.14 \mathrm{~K} / \mathrm{d}$ ), but the magnitude of the error is still very small when compared to the high absolute values of HR in the upper atmosphere (global HR value of topmost layer: $\sim 10.7 \mathrm{~K} / \mathrm{d}$ ). We have not been able to find a convincing explanation for this behavior, but one of our hypotheses is that it may be related to the new two-stream PPH solutions for diffuse incidence (which in this case mostly comes from the layers below) implemented in the GWTSA version of the CORAM for clear skies. The remaining features in Fig. 8 show the expected behaviour with larger HR errors in the $\mathrm{SH}$ than the $\mathrm{NH}$, due to higher insolation, $\mathrm{PPH}$ overestimates high in the atmosphere paired with underestimates in the lower atmosphere, and overall improvements (except for the very top layers as previously mentioned) when the GWTSA is introduced.

\section{LW experiments}

The LW CORAM was only used unmodified with ICA and PPH runs performed using the methodology described above for the SW experiments. Note that in the ICA 
calculations the subgrid variability of temperatures had a negligible influence on the results (echoing similar results by Fu et al., 2000) and was ignored. The broadband surface emissivities of Räisänen (1999) were used for these runs and surface temperatures were set equal to the average temperature of the lowest CRM layer (CAM surface temperatures were not available). Again, the ozone profile of a midlatitude summer atmosphere was used in all gridboxes.

The figures summarizing the results are similar to those shown previously for the SW results: Figure 9 shows the globally averaged mean errors and rms errors of PPH for all gridboxes and for overcast ("ovc") gridboxes only, for the following quantities: outgoing LW radiation (OLR), net flux absorbed at the surface (NSFC), and net flux absorbed within the atmosphere (ATMA). Figure 10 shows the zonally-averaged results for the same quantities. Figure 11 shows the globally averaged LW cooling rates. (COOLRs) for ICA and PPH (left) and the PPH rms errors (right), and Figure 12 shows zonally-averaged PPH COOLR errors. Results are again daily-averaged over all 24 snapshots, and positive mean error values indicate overestimate relative to the ICA.

It is immediately apparent that the results are consistent with the conventional wisdom that cloud water horizontal inhomogeneity is less important in the $\mathrm{LW}$ than in the SW. Global errors for OLR and ATMA are $\sim 2 \mathrm{Wm}^{-2}$ (underestimates, as in Fu et al., 2000 , albeit quite lower than their cirrus-specific value of $\sim 14 \mathrm{Wm}^{-2}$ ), and the NSFC error is almost zero, although this is the result of canceling errors since the rms error magnitude exceeds $2 \mathrm{Wm}^{-2}$ (additional evidence of the fortuitous cancelling in the global average is provided in Fig. 10 which reveals a latitudinal structure in the errors). In contrast to the SW, but still not unexpectedly, the errors are more symmetric with respect 
to the equator which is again the area characterized by the largest errors (Fig. 10). Errors in the COOLR are very small for global averages (Fig. 11) and have a zonal distribution (Fig. 12) somewhat more difficult to associate with the cloud properties distribution of Fig. 1 than in the SW. The largest cooling overestimates occur around the tops of the deep convective clouds of the ITCZ (consistent with the convex non-linear dependence of cloud emissivity on optical depth). For the middle layers of the atmosphere, the net imbalance of PPH fluxes yields underestimates of the cooling in most regions, the end result of neglecting inhomogeneity for upward and downward cloud emissions.

\section{Conclusions}

The results shown in this paper exemplify the generally good performance of the SW and LW CORAMs by Chou and collaborators, currently implemented in a variety of GSFC GCMs, when mean profiles of heterogeneous cloud fields from a super parameterization experiment with an atmospheric GCM are used as input in radiative transfer calculations. In the SW case, with no subgrid variability information provided, but with perfect profiles of mean water path and cloud fraction as input, the global mean errors, with respect to ICA, of the original PPH-like code do not exceed $4 \mathrm{Wm}^{-2}$ for fluxes at the atmospheric boundaries and $0.3 \mathrm{Wm}^{-2}$ for the flux absorbed within the atmosphere. Zonal HR errors are always below $0.3 \mathrm{~K} / \mathrm{d}$ with overestimates for high clouds and underestimates for low clouds. Zonal flux errors are larger in the tropics (reaching $\sim 10$

$\mathrm{Wm}^{-2}$ ) where the thickest and most inhomogeneous clouds are encountered, and larger in the $\mathrm{SH}$ than in the $\mathrm{NH}$, mainly because of the greater available solar energy. PPH overestimates reflected flux and underestimates by almost the same amount the flux 
absorbed at the surface, therefore producing very small errors for the flux absorbed by the atmosphere. When the scaling of optical depth and the overlap assumptions associated with it are removed and the random overlap assumption applied everywhere, the deterioration in performance is notable. On the other hand, implementation of the GWTSA yields substantial improvements in overall performance. Also, GWTSA has significantly lower rms errors than the other two approximations, indicating that cancellation of errors when spatial averages are taken is less extensive.

In the LW case, PPH underestimates OLR and absorbed flux in the atmosphere by equal amounts $\left(\sim 2 \mathrm{Wm}^{-2}\right.$ at global scales), and is almost perfect for NSFC (although this is the net result of overestimates in low and underestimates in high latitudes). Again, the largest flux errors for OLR and ATMA occur in the tropics. The COOLRs tend to be overestimated by the PPH at the higher levels of the troposphere and underestimated at lower levels with errors always less than $0.6 \mathrm{~K} / \mathrm{d}$.

These results suggest that previous studies demonstrating the inadequacy of planeparallel models may have unfairly focused on worst scenario cases, and that current radiative transfer algorithms of GCMs may be more capable than previously thought in estimating realistic spatial and temporal averages of radiative fluxes, as long as they are provided with realistic cloud profiles. It would therefore be useful to conduct a future study to determine whether the encouraging results shown here retain their quality when a larger set of snapshots from such super parameterization experiments is used. Another good test for the CORAMs expected to be feasible in the near future would be to use as input cloud profiles from the upcoming CLOUDSAT and CALIPSO missions (Stephens et al., 2002). 
Given the consistently better performance of the GWTSA in the SW CORAM, a strategy for addressing the issues that would allow its operational implementation in a GCM should be seriously contemplated. The most challenging of these issues will probably be prognosing or diagnosing $v$ for each cloudy layer. Finally, one must keep in mind that even if the errors of the particular SW and LW CORAMs are small, the fact that they appear to be systematic (even for the GWTSA) implies that they may be potentially important climatically; their impact can be fully assessed only with GCM climate simulations.

Acknowledgements: We would like to thank Petri Räisänen for providing the surface albedos and emissivities. This work was supported by NASA grant NAG5-11631 and the U. S. Department of Energy, under grant DE-AI02-00ER62939, as part of the Atmospheric Radiation Measurement Program. 


\section{Figure Captions}

Figure 1 Daily-averaged zonal values of layer cloud fraction, water path, and inhomogeneity parameter $v$.

Figure 2 Sketch of how cloud overlap and optical thickness scaling is implemented in the SW CORAM (from Chou et al. 1998).

Figure 3 Daily-averaged global SW flux errors for all or just overcast (“ovc”) gridboxes. Figure 4 Daily-averaged global SW flux errors for gridboxes with mean (cloudy subcolumns only) atmospheric column water path greater $200 \mathrm{gm}^{-2}$ ("1") and gridboxes with $v$ of integrated water path less than 1.5 (“2”).

Figure 5 Daily-averaged zonal SW flux mean errors for RTOA (top) and corresponding rms errors (bottom).

Figure 6 RTOA PPH random and GWTSA errors vs. PPH errors for the entire portion of the globe receiving solar illumination. Each point is the daily average of a gridbox.

Figure 7 Daily-averaged global SW heating rates (left) and rms errors for the three approximate methods (right).

Figure 8 Daily-averaged zonal SW heating rate errors. Top: PPH; middle: PPH random; bottom: GWTSA.

Figure 9 Daily-averaged global LW flux errors of PPH.

Figure 10 Daily-averaged zonal LW flux mean errors of PPH for OLR, NSFC, ATMA (top), and corresponding rms errors.

Figure 11 Daily-averaged global LW cooling rates (left) and rms errors for PPH (right). Figure 12 Daily-averaged zonal LW cooling rate errors of PPH. 


\section{References}

Barker, H. W., 1996. A parameterization for computing grid-averaged solar fluxes for inhomogeneous marine boundary layer clouds. Part I: Methodology and homogeneous biases. J. Atmos. Sci. 53, 2289-2303.

Barker, H. W., G. L. Stephens, and Q. Fu, 1999. The sensitivity of domain-averaged solar fluxes to assumptions about cloud geometry. Q. J. R. Meteorol. Soc. 125, 2127-2152.

Barker, H. W., and Q. Fu, 1999. Modelling domain-averaged solar fluxes for an evolving tropical cloud system. Atmos. Ocean. Opt. 12(3), 211-217.

Barker, H. W., and Q. Fu, 2000. Assessment and optimization of the gamma-weighted two-stream approximation. J. Atmos. Sci. 57, 1181-1188.

Barker, H. W., and 31 coauthors, 2002. Assessing 1D atmospheric solar radiative transfer models: interpretation and handling of unresolved clouds. J. Clim., in press.

Cahalan, R. F., W. Ridgway, W. J. Wiscombe, T. L. Bell and J. B. Snider, 1994. The albedo of fractal stratocumulus clouds. J. Atmos. Sci. 51, 2434 -2455.

Cairns, B., A. A. Lacis, and B. E. Carlson, 2000. Absorption within inhomogeneous clouds and its parameterization in general circulation models. J. Atmos. Sci. 57, 700714.

Chou, M.-D., M. J. Suarez, C.-H. Ho. M. M.-H. Yan, and K.-T. Lee, 1998. Parameterizations for cloud overlapping and shortwave single-scattering properties for use in general circulation and cloud ensemble models. J. Clim. 11, 202-214.

Chou, M.-D., and M. J. Suarez, 1999. A shortwave radiation parameterization for atmospheric studies. NASA Tech. Memo. 15(104606), 42 pp.

Chou, M.-D., M. J. Suarez, X.-Z. Liang, and M. M.-H. Yan, 2001. A thermal infrared 
radiation parameterization for atmospheric studies. NASA Tech. Memo. 19(104606), $55 \mathrm{pp}$.

Chou, M.-D., K.-T. Lee, and P. Yang, 2002. Parameterization of shortwave cloud optical properties for a mixture of ice particle habits for use in atmospheric models, J. Geophys. Res. 107(D21), doi:10.1029/2002JD002061, AAC 22-1- AAC 22-9.

Fu, Q., B. Carlin, and G. Mace, 2000. Cirrus horizontal inhomogeneity and OLR bias. Geophys. Res. Lett. 27, 3341-3344.

Harshvardhan, D. A. Randall, and T. G. Corsetti, 1987. A fast radiation parameterization for atmospheric circulation models. J. Geophys. Res. 92, 1009-1016.

Kato, S., 2003. Computation of domain-averaged shortwave irradiance by a onedimensional algorithm: Incorporating correlations between optical thickness and direct incident radiation. J. Atmos. Sci. 60, 182-193.

Khairoutdinov, M. F., and D. A. Randall, 2003. Cloud resolving modeling of the ARM Summer 1997 IOP: Model formulation, results, uncertainties and sensitivities. J. Atmos. Sci. 60, 607-625.

Khairoutdinov, M. F., D. A. Randall, and C. DeMott, 2003. Simulations of the atmospheric general circulation using a Cloud-Resolving Model and a superparameterization of physical processes. J. Atmos. Sci., submitted.

Li, J., H. W. Barker, 2002. Accounting for unresolved clouds in a ID infrared radiative transfer model. Part II: Horizontal variability of cloud water path. J. Atmos. Sci. 59, $3321-3339$.

McFarquhar, G. M., 2001. Comments on "Parametrization of effective sizes of cirruscloud particles and its verification against observations" by Z. Sun, and L. Rikus, Q. 
J. R. Meteorol. Soc. 127, 261-266.

Meador, W. E., and W. R. Weaver, 1980. Two-stream approximations to radiative transfer in planetary atmospheres: A unified description of existing methods and a new improvement. J. Atmos. Sci. 37, 630-643.

Oreopoulos, L., and H. W. Barker, 1999. Accounting for subgrid-scale cloud variability in a multi-layer 1D solar radiative transfer algorithm. Q. J. R. Meteorol. Soc. 126, 301-330.

Räisänen, P., 1999. Effect of vertical resolution on cloudy-sky radiation calculations: Tests with two schemes. J. Geophys. Res. 104, 27407-27419.

Räisänen, P., H. W. Barker, M. F. Khairoutdinov, J. Li, and D. A. Randall, 2003. Stochastic generation of subgrid-scale cloudy columns for large-scale models. Q. J. R. Meteor. Soc., submitted,

Rossow, W. B., C. Delo, and B. Cairns, 2002. Implications of the observed mesoscale variations of clouds for the Earth's radiation budget. J. Clim. 15, 557-585.

Stephens, G. L., D. G. Vane, R. J. Boain, G. G. Mace, K. Sassen, Z. Wang, A. J. Illingworth, E. J. O’Connor, W. B. Rossow, S. L. Durden. S. D. Miller, R. T. Austin, A. Benedetti, C. Mitrescu, and the CloudSat Science Team, 2002. The CloudSat mission and the A-Train: A new dimension of space-based observations of clouds and precipitation. Bull. Amer. Meteor. Soc. 83, 1771-1790.

Szczap, F., H. Isaka, M. Saute, B. Guillemet, A. Ioltukhovski, 2000a. Effective radiative properties of bounded cascade nonabsorbing clouds: Definition of the equivalent homogeneous cloud approximation. J. Geophys. Res. 105 , 20617-20633.

Szczap, F., H. Isaka, M. Saute, B. Guillemet, A. Ioltukhovski, 2000b. Effective radiative properties of bounded cascade nonabsorbing clouds: Definition of an effective single- 
scattering albedo. J. Geophys. Res. 105 , $20635-20648$.

Szczodrak, M., P. H. Austin, P. B. Krummel, 2001. Variability of optical depth and effective radius in marine stratocumulus clouds. J. Atmos. Sci. 58, 2912-2926.

Tian, L. and J. A. Curry, 1989. Cloud overlap statistics. J. Geophys. Res. 94, 9925-9935. 

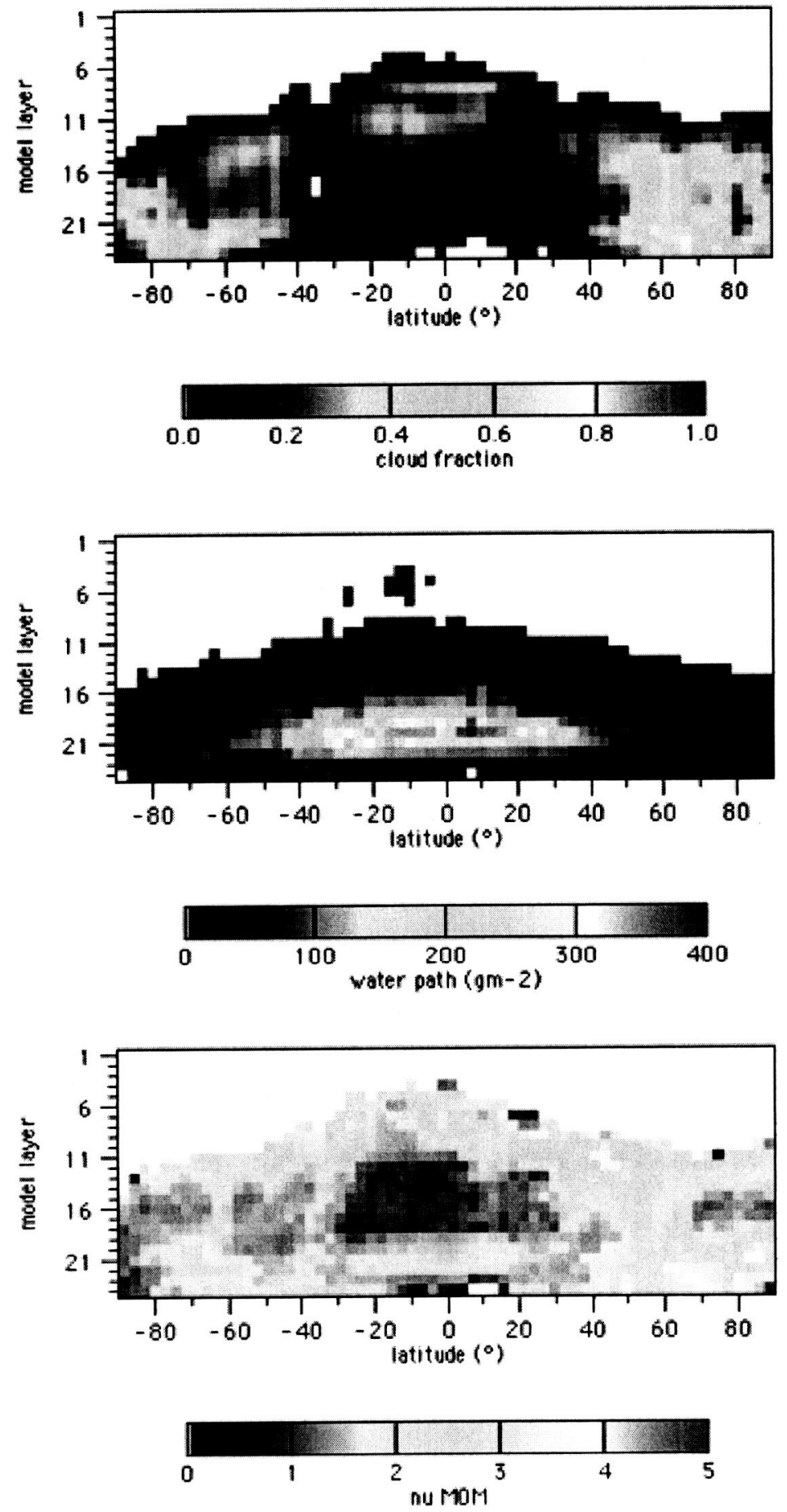

Figure 1 Daily-averaged zonal values of layer cloud fraction, water path, and inhomogeneity parameter $v$. 


\section{Cloud Overlapping}

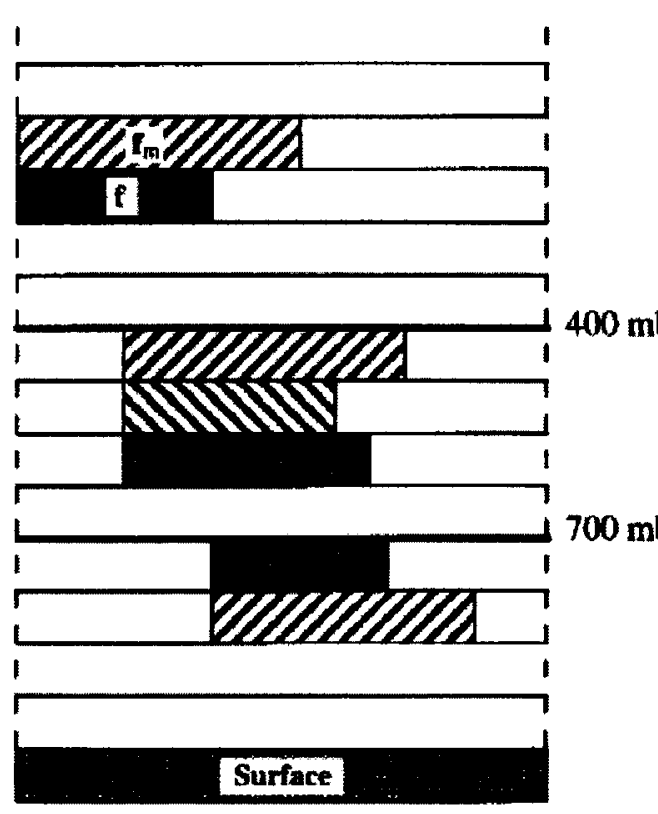

Optical-thickness Scaling

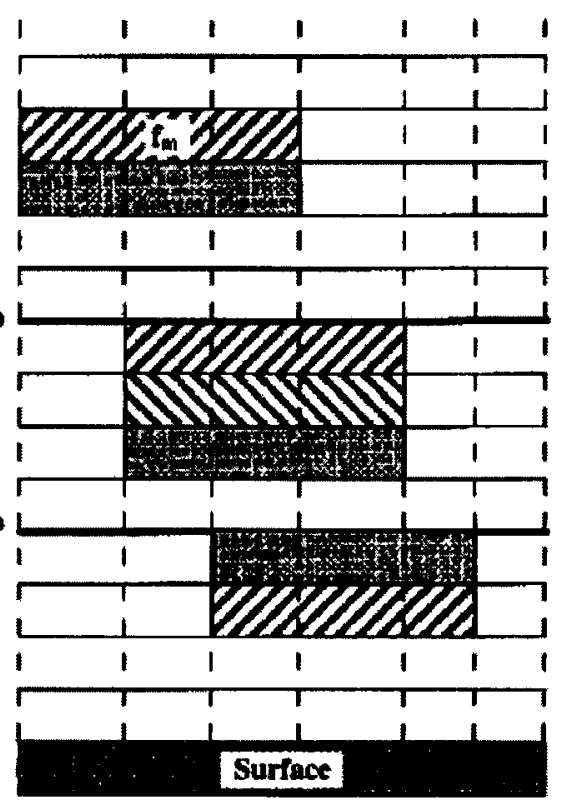

Figure 2 Sketch of how cloud overlap and optical thickness scaling is implemented in the SW CORAM (from Chou et al. 1998). 


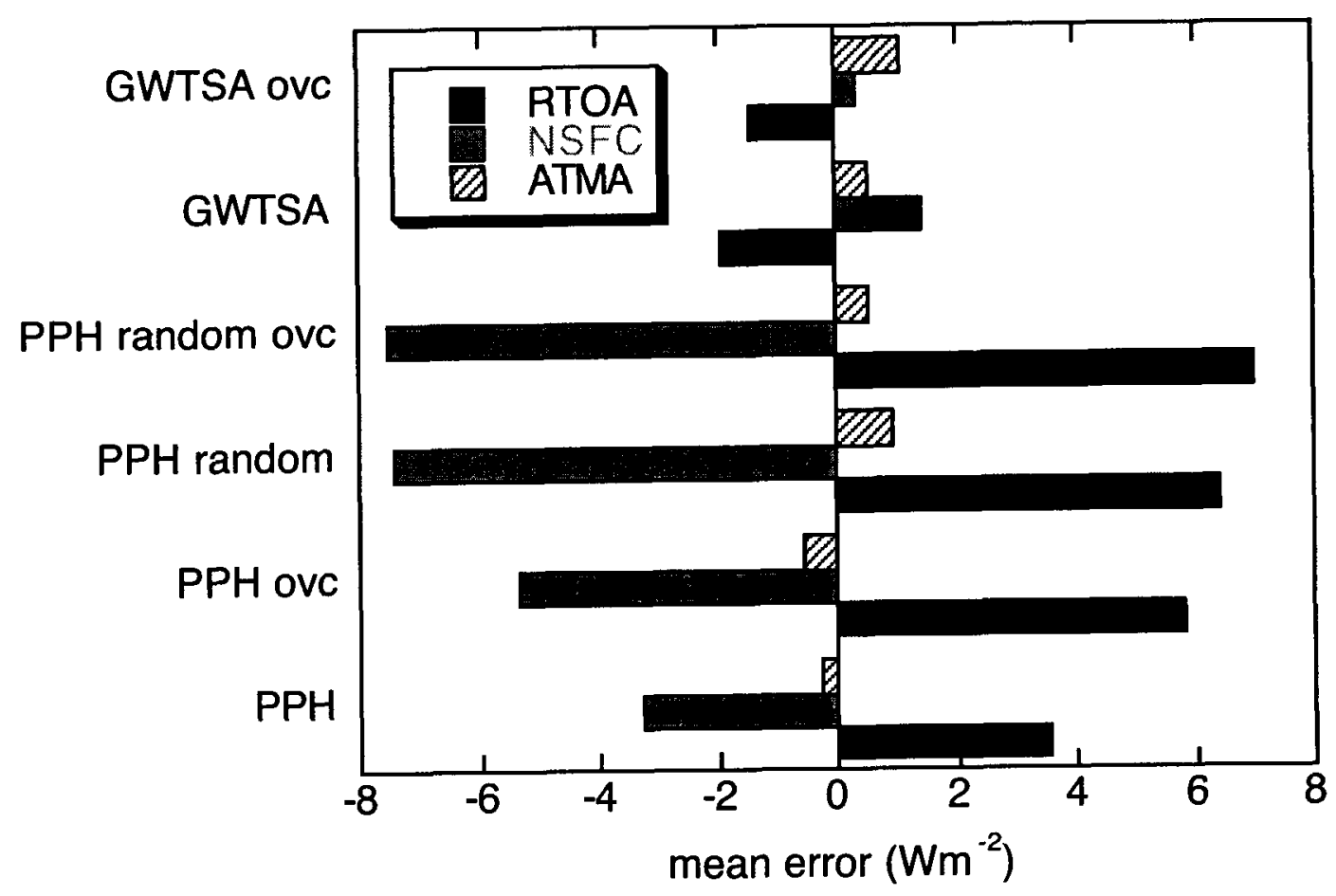

Figure 3 Daily-averaged global SW flux errors for all or just overcast ("ovc") gridboxes. 


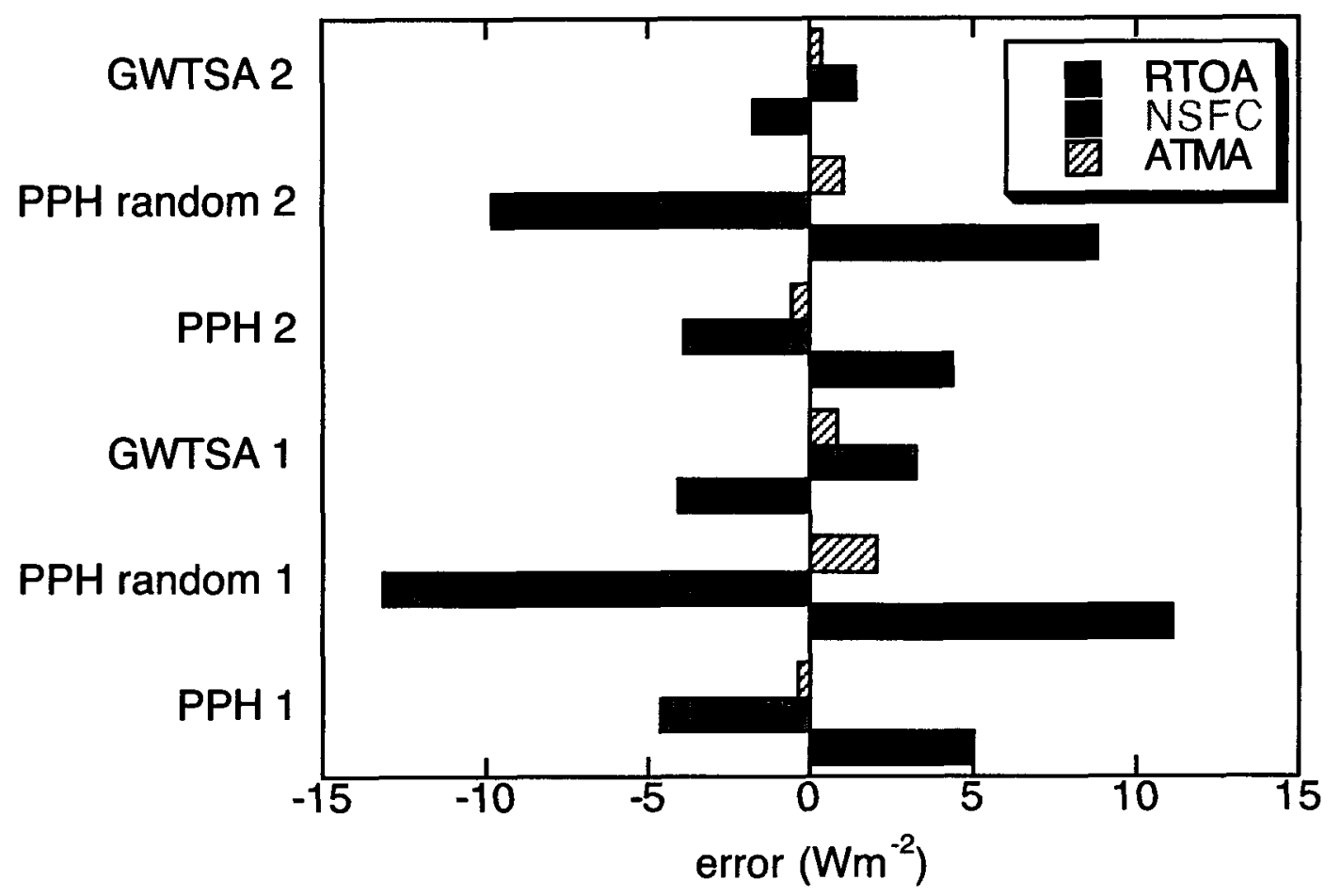

Figure 4 Daily-averaged global SW flux errors for gridboxes with mean (cloudy subcolumns only) atmospheric column water path greater $200 \mathrm{gm}^{-2}$ ("1") and gridboxes with $v$ of integrated water path less than 1.5 ("2"). 

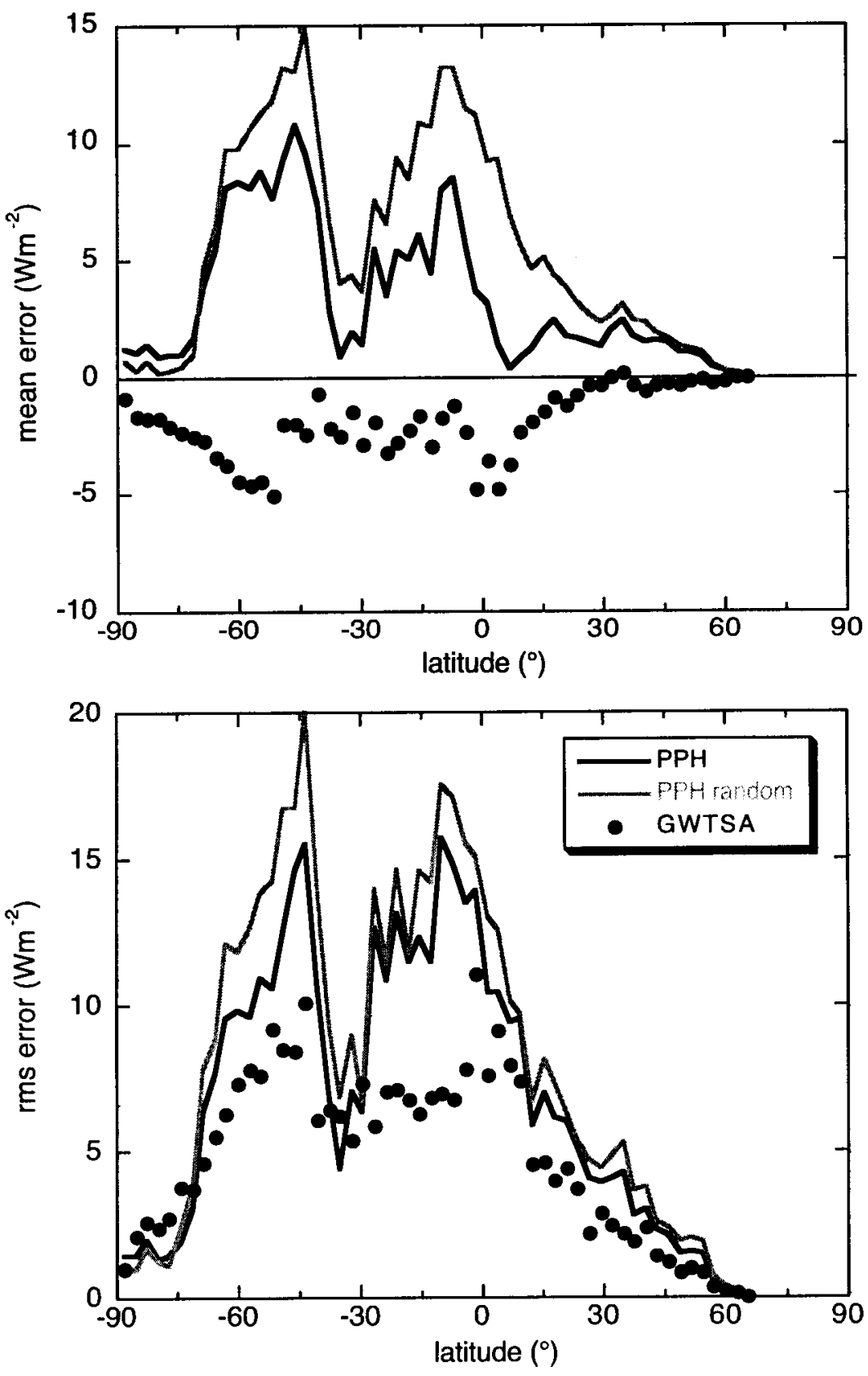

Figure 5 Daily-averaged zonal SW flux mean errors for RTOA (top) and corresponding rms errors (bottom). 


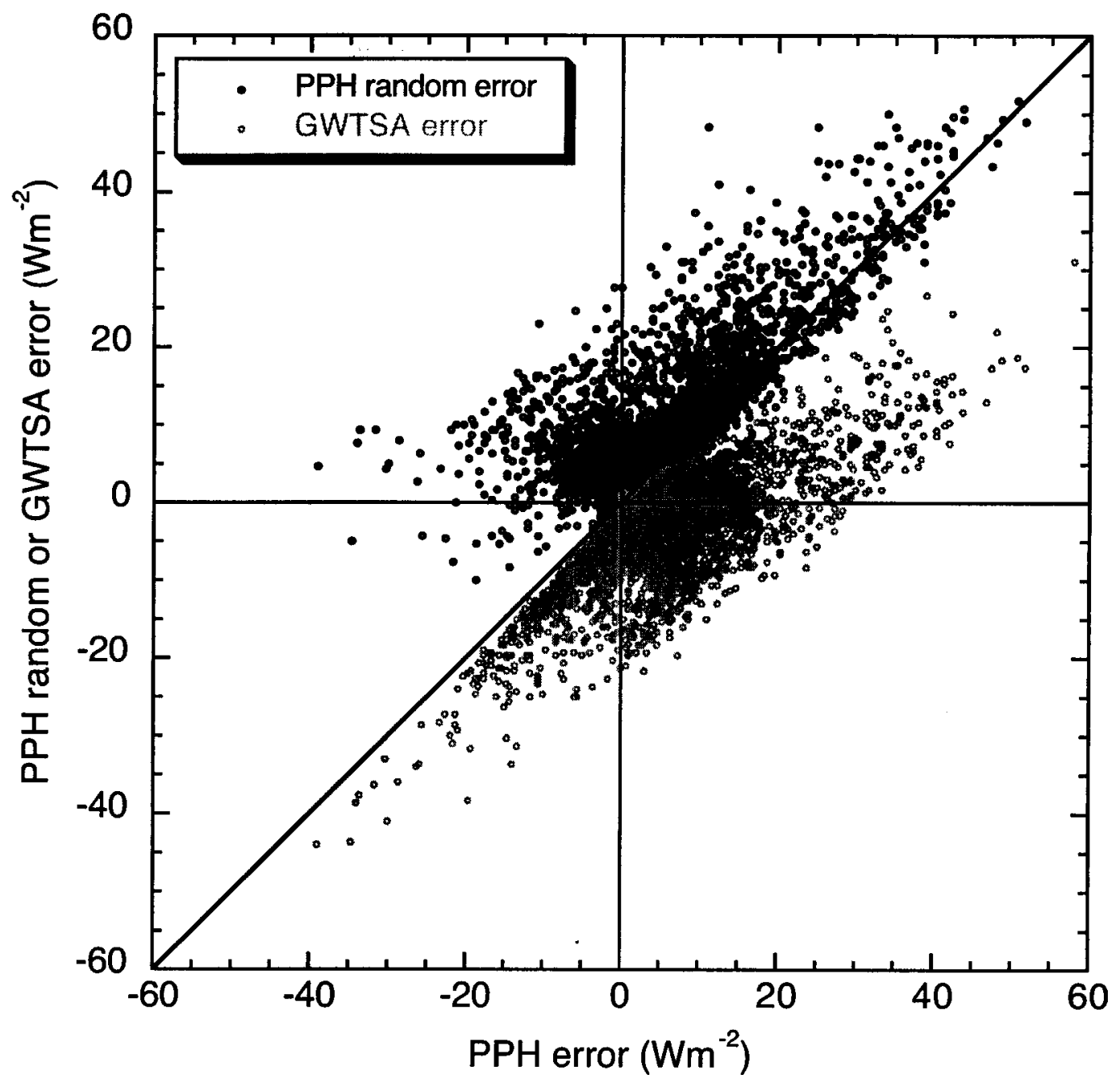

Figure 6 RTOA PPH random and GWTSA errors vs. PPH errors for the entire portion of the globe receiving solar illumination. Each point is the daily average of a gridbox. 

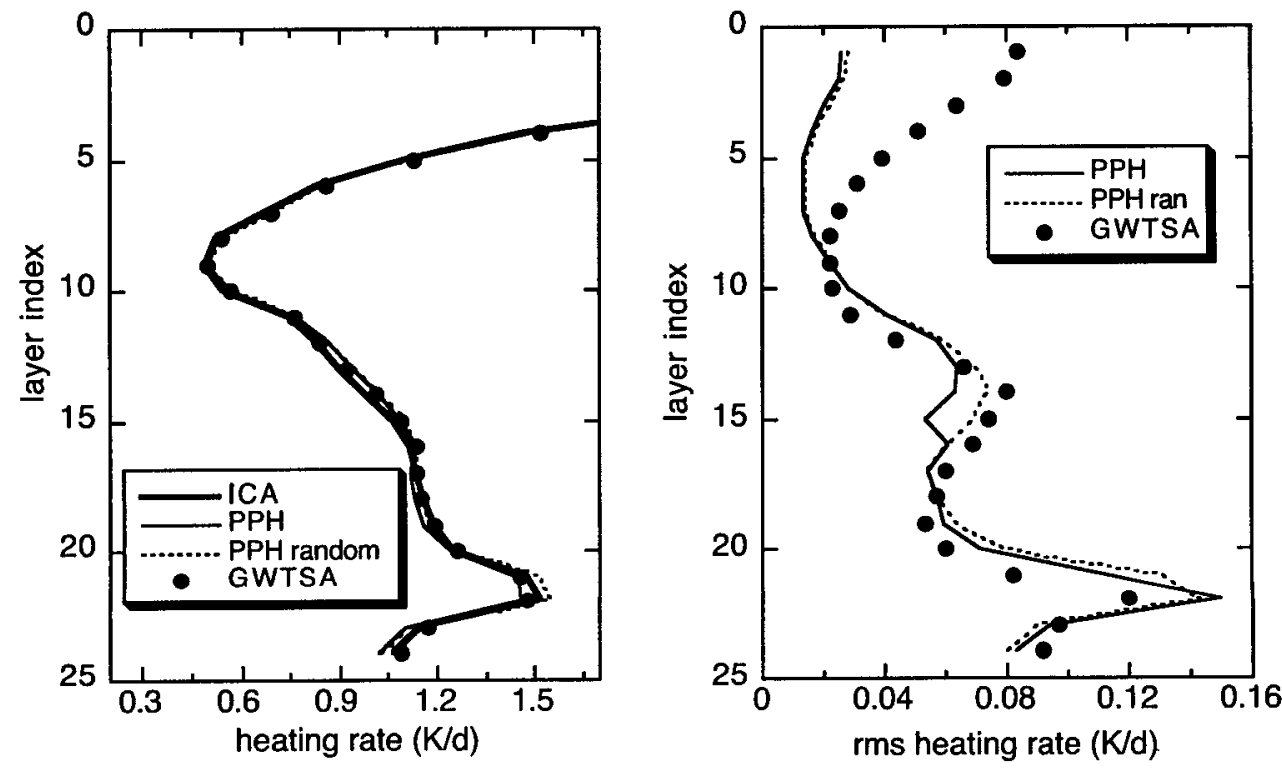

Figure 7 Daily-averaged global SW heating rates (left) and rms errors for the three approximate methods (right). 

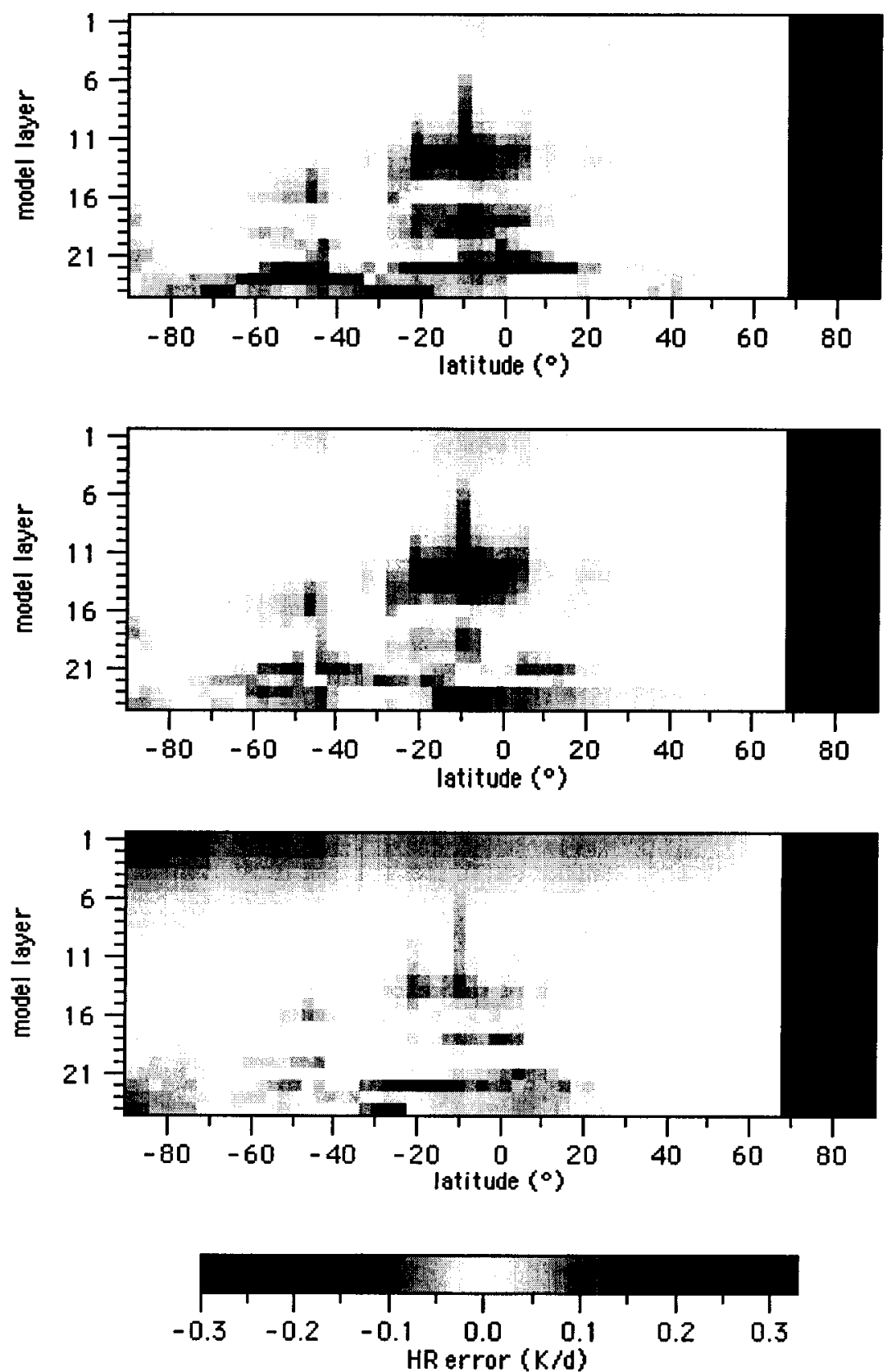

Figure 8 Daily-averaged zonal SW heating rate errors. Top: PPH; middle: PPH random; bottom: GWTSA. 


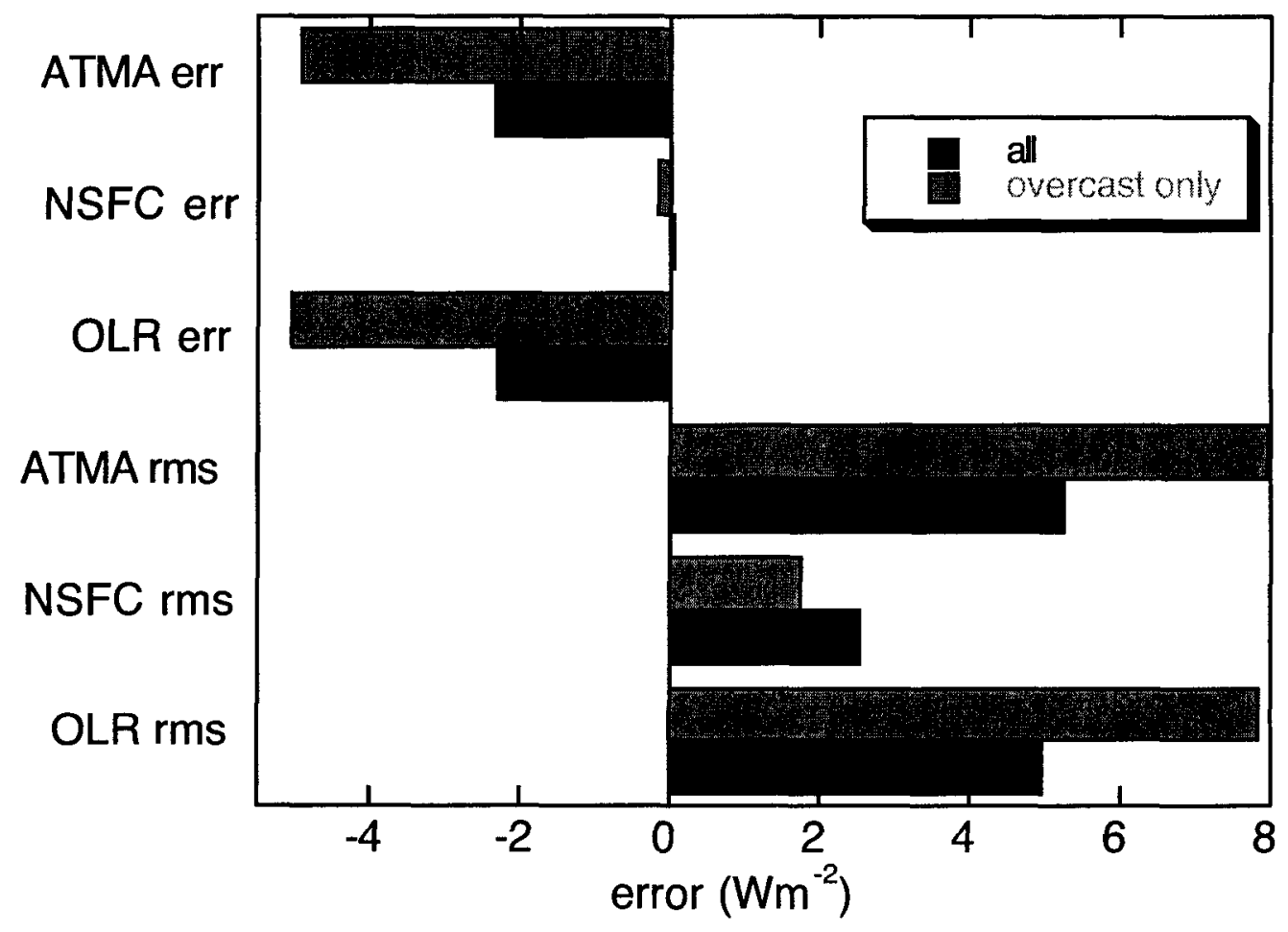

Figure 9 Daily-averaged global LW flux errors of PPH. 

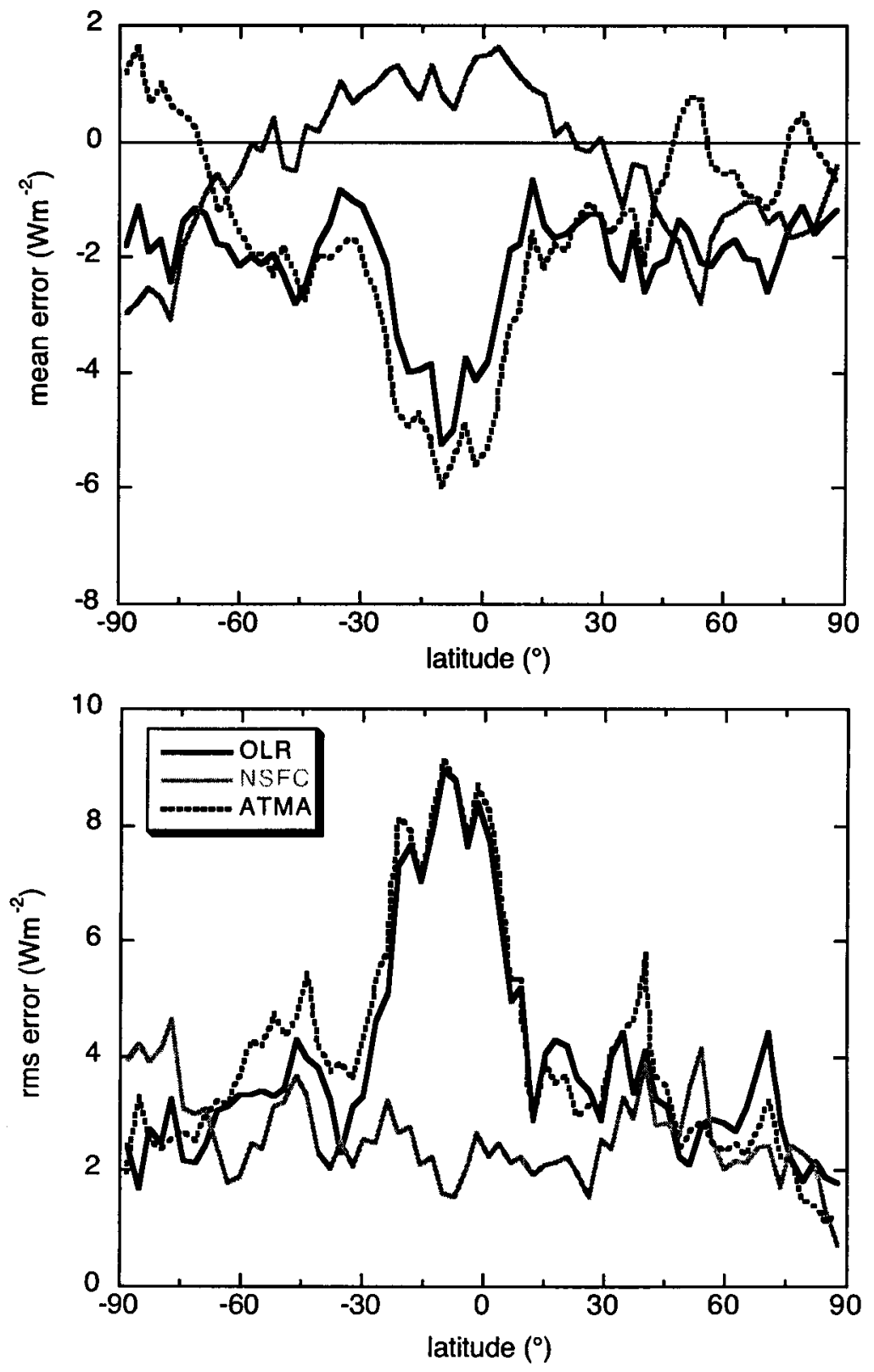

Figure 10 Daily-averaged zonal LW flux mean errors of PPH for OLR, NSFC, ATMA (top), and corresponding rms errors. 

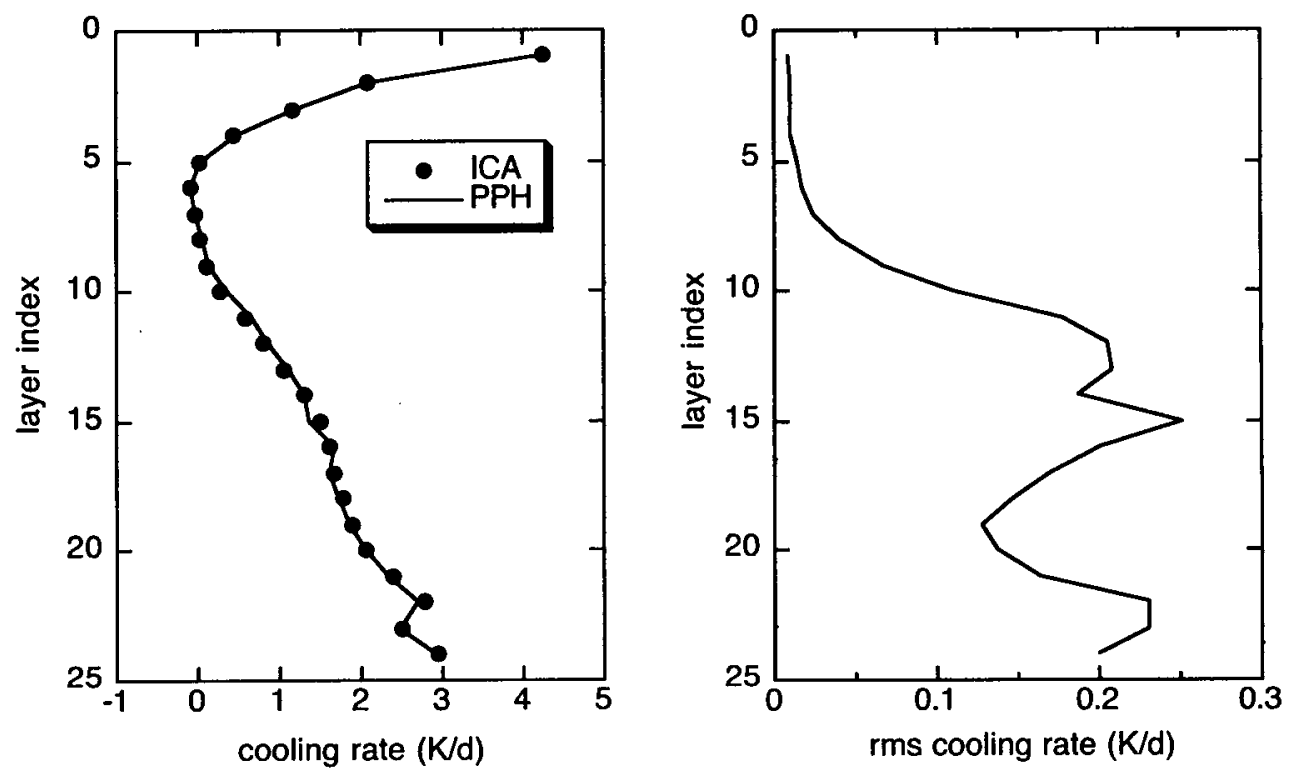

Figure 11 Daily-averaged global LW cooling rates (left) and rms errors for PPH (right). 

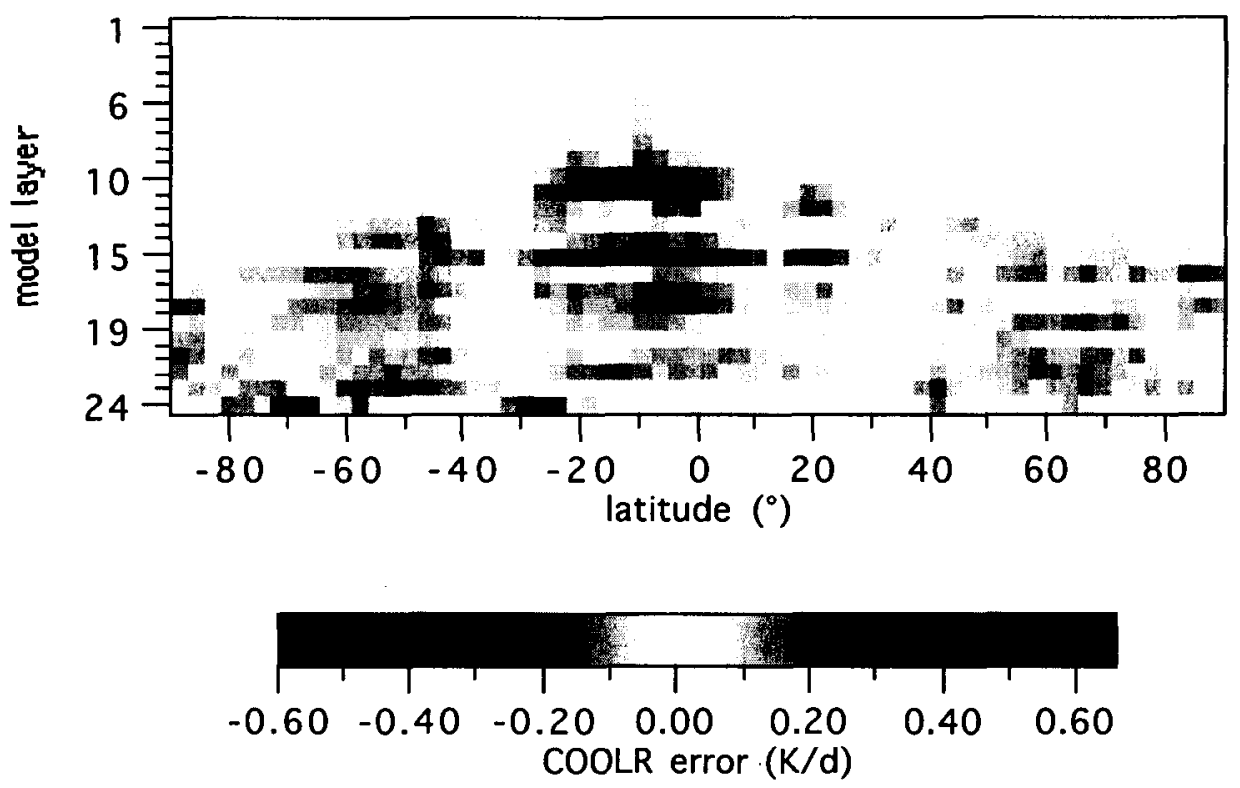

Figure 12 Daily-averaged zonal LW cooling rate errors of PPH. 